\title{
COMPUTERSIMULATION ZUR VERIFIZIERUNG UND PRÄSENTATION EINES AUTOMATISCH GESTEUERTEN MAMMA-BIOPSIESYSTEMS
}

\author{
M. Dorsch ${ }^{1}$, J. Oellinger ${ }^{1}$, O. Wendt ${ }^{2}$, U. Boenick $^{2}$ \\ ${ }^{1}$ Universitätsklinikum Charité, Campus Virchow-Klinikum, Strahlen- und Poliklinik \\ ${ }^{2}$ Technische Universität Berlin, Institut für Mikrotechnik und Medizintechnik
}

medtech@bmt1.kf.TU-Berlin.DE

\section{EINLEITUNG}

Die computerunterstützte Simulations- und Animationstechnik ist ein allgemein anerkanntes Hilfsmittel bei der Planung und Realisierung von technischen Systemen. Der Schwerpunkt des Simulations- und Animationseinsatzes lag ursprünglich nur auf der Planungsabsicherung. Zunehmend wird die Simulation und Animation durchgängig in allen Phasen des Planungs- und Realisierungsprozesses genutzt [1].

In einem Forschungsprojekt wurde eine neuartige automatisch gesteuerte Einrichtung zur präzisen Navigation medizinischer Instrumente innerhalb von MRTomographen, insbesondere zur histologischen Sicherung von Kleintumoren, entwickelt. Die gesamte Einrichtung läßt sich gemeinsam mit einem Patienten im Untersuchungsbereich eines MR-Tomographen positionieren, wie prinzipiell in Abb.1 gezeigt. Mittels einer 3D-Simulation wie auch Animation konnte eine Überprüfung und Optimierung des komplexen Gesamtsystems, d.h. der einzelnen Systemkomponenten und deren kinematischen Bewegungsabläufe erfolgen.

Desweiteren wurde damit eine Grundlage für die Erstellung einer Präsentationsvorlage realisiert, um der Öffentlichkeit die einzelnen Ablaufvorgänge einfach und plausibel darzustellen und zukünftige Anwender in dieses System schnell einzuweisen.

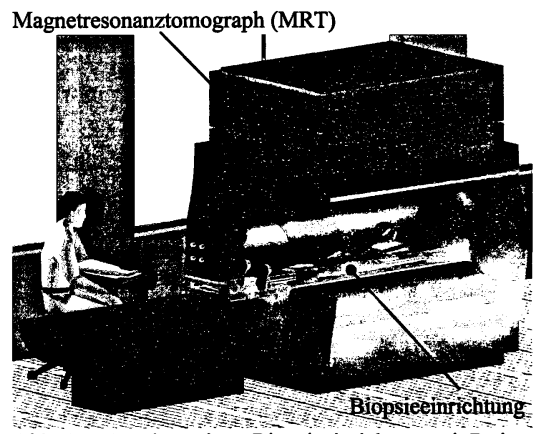

Abb. 1: MRT-integrierte Biopsieeinrichtung mit Patientin in der Untersuchungsposition

\section{MATERIALIEN UND METHODEN}

Die erstellten 2D-Zeichnungen (AutoCAD ${ }^{\circledR} \mathrm{R} 14$, AutoDesk) werden mittels unterschiedlicher Modifikatoren und Operatoren, wie z.B. der Extrusion oder der Boole'schen Algebra zu 3D-Objekten umgewandelt, damit sie nachfolgend in das Simulations- und Animationsprogramm 3D Studio MAX ${ }^{\circledR} \mathrm{R} 2.5$ (Kinetix) mittels einer Exportfunktion eingebunden werden können. Im 3D Studio Max R 2.5 werden die einzelnen 3D-Objekte zu Teilsystemen zusammengesetzt und in einem ersten Schritt die geometrischen Dimensionen und Lagekoordinaten sowohl der einzelnen Objekte als auch der Teilsysteme überprüft. Hierbei können unter Zuhilfenahme verschiedener Modifikatoren unter anderem verschiedene Beleuchtungsmodelle, wie z.B. das „Phong'sche“ programmiert werden, das zur Be-rechnung eines Bildes die optischen Brechungs- und Reflexionseigenschaften von Objektoberflächen mit be-rücksichtigt [2]. Daneben können die Objekte auch mit realen Farben und 3DTexturen versehen werden. Über entsprechende Kontrollfunktionen werden für die einzelnen kinematischen Bewegungsabläufe und zeitlichen Abfolgen der einzelnen Teilsysteme „Eltern-Kind-Beziehungen“ und Hierarchiestrukturen erstellt. Für die Erstellung der menschlichen Charaktere wurde ein Plug-In, Character Studio $^{\circledR}$ R 2.0 (Firma Kinetix), eingesetzt. Damit konnte eine komplette Skelettstruktur mit Muskulatur und Sehnen aufgebaut und so dem realen menschlichen Bewegungsablauf optimal angepaßt werden. Mit Hilfe eines Renderers werden die erstellten Szenen mit ihren Bewegungs- und zeitlichen Abläufen unter anderem als Videosequenzen gespeichert, die letztendlich in gängigen Präsentationsprogrammen ihre Anwendung finden.

\section{ERGEBNISSE}

Prinzipiell läßt sich die Simulation in zwei Teilbereiche untergliedern. Zum einen in den Magnetresonanztomographen mit Patiententisch, Patientin und Arzt, siehe Abb. 1, und zum anderen in das eigentliche Biopsiesystem mit seinen Teilkomponenten, auf das besonders Wert gelegt wurde. So besteht das Biopsiesystem selbst aus einem Rahmengestell, was exemplarisch in Abb. 2 dargestellt ist, mit einer integrierten MRSende- und Empfangsdoppelspule, sowie einem Fixie- 
rungssystem zur Aufnahme und Stabilisierung der Mammae. Zudem werden einzelne Komponenten zur Navigation und Anwendung eines medizinischen Instrumentes, bestehend aus mehreren speziellen Antriebseinheiten und Führungselementen, benützt.

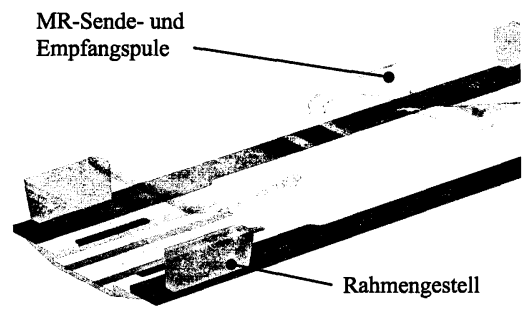

Abb. 2: Rahmengestell

So war es durch die hohe Detailgenauigkeit und der dreidimensionalen Darstellung möglich, z.B. hinsichtlich des Designs / Ergonomie oder auch der Funktionalität, frühzeitig Änderungen an den einzelnen Objekten durchzuführen. Zudem stellte sich heraus, ob sich einzelne Objekte während der kinematischen Bewegungsabläufe untereinander berühren und somit $\mathrm{zu}$ ungewollten Kollisionen führen. Ferner konnte mit Hilfe der 3D-Simulation der Arbeitsraum sowohl des medizinischen Instruments als auch des Arztes bzw. der Patientin festgelegt werden. Überdies konnten auch unter anderem die maximal einstellbaren Biopsiewinkel des medizinischen Instrumentes bestimmt werden, die für eine anschließende Automatisierung von entscheidender Bedeutung sind.

In der nachfolgenden Abbildung 3 ist beispielhaft ein Spülsystem zur Entfernung von z.B. Gewebeproben dargestellt. Die sich auf dem medizinischen Instrument befindliche Gewebeprobe wird über einen Spülkreislauf aus dem Biopsiesystem herausbefördert und konserviert. Der Weg der Spül- und Konservierungsflüssigkeit ist anhand der Pfeile dargestellt.

Mit Hilfe der 3D-Simulation konnten mögliche Anschlüsse für Schläuche und deren Verlegung hauptsächlich hinsichtlich minimaler Schlauchlängen optimiert werden. Außerdem wurde überprüft, ob Kollisionen mit anderen Objekten auftreten oder Schläuche bzw. Kabel auf Grund ihrer räumlichen Bewegungen eingeklemmt werden.

In der abschließenden Tab. 1 sind allgemeine Kriterien zur Bewertung von technischen 3D-Simulationen gegenüber realen Objekten und Systemen aufgeführt.

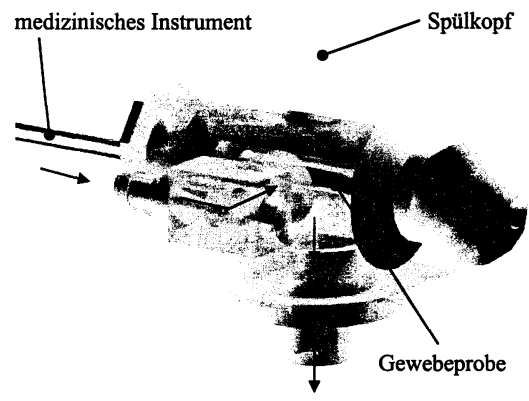

Abb. 3: Spülsystem

Tab. 1: Mögliche Kriterien zur Bewertung von technischen 3D-Simulationen

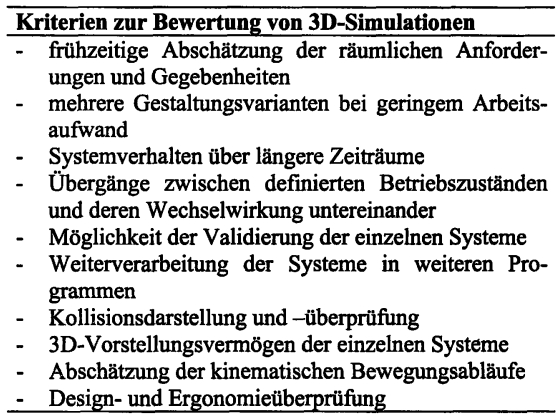

\section{SCHLUBFOLGERUNGEN}

Trotz der hervorragenden Darstellungsmöglichkeiten stellen 3D-Simulationen immer nur Approximationen an das reale System dar. Sie können unter anderem keine Fertigungstoleranzen, sowie werkstoffspezifische Eigenschaften, wie z.B. mechanische, thermische oder elektrische Eigenschaften, im einzelnen berücksichtigen, da sich der damit verbundene (Zeit-)Aufwand nicht mehr rentieren würde. Die Simulatortechnologie stellt jedoch eine gute Möglichkeit für die drei-dimensionale Überprüfung von geometrischen Problemstellungen und kinematischen Abläufen dar. Zudem kann sie als Präsentationsvorlage einer interessierten Öffentlichkeit komplexe Systeme plausibel vermitteln.

\section{LITERATURHINWEISE}

[1] VDI-Richtlinie 3633

[2] K.D. Tönnies, H.U. Lemke, 3D-Computergrafische Darstellungen, Oldenbourg, München, Wien 1994 